\title{
Effects of the Nonsteroidal Anti-inflammatory Drug Celecoxib on Mitochondrial Function
}

\author{
Yohei Tatematsu, ${ }^{a}$ Haruhi Fujita, ${ }^{a}$ Hiroki Hayashi, ${ }^{a}$ Atsushi Yamamoto, ${ }^{a}$ Atsushi Tabata, ${ }^{b}$ \\ Hideaki Nagamune, ${ }^{b}$ and Kazuto Ohkura*,a \\ ${ }^{a}$ Graduate School of Pharmaceutical Sciences, Suzuka University of Medical Science; Suzuka, Mie 513-8670, \\ Japan: and ${ }^{b}$ Department of Bioengineering, Division of Bioscience and Bioindustry, Graduate School of Technology, \\ Industrial and Social Sciences, Tokushima University; Tokushima 770-8513, Japan. \\ Received July 3, 2017; accepted December 1, 2017
}

\begin{abstract}
Nonsteroidal anti-inflammatory drugs (NSAIDs) are widely used to treat inflammation and pain. In the present study, we examined the effects of celecoxib, a cyclooxygenase-2 (COX-2)-selective NSAID, on rat liver mitochondrial function. Celecoxib dose-dependently induced mitochondria swelling, which was not suppressed by cyclosporine A (CsA). The oxygen consumption rate in mitochondria-suspended solution was facilitated by the addition of celecoxib, and its uncoupling activity was observed. Celecoxib also suppressed SF6847-induced uncoupling, and appeared to exert inhibitory effects on the electron transport chain. Celecoxib suppressed the state 3 oxygen consumption rate in the presence of ADP. Protein release from the mitochondrial matrix was detected following the addition of celecoxib, and aldehyde dehydrogenase 2 (ALDH2) and hydroxymethylglutaryl-CoA (HMG-CoA) synthase 2 (HMGCS2) bands were confirmed in a Western blot analysis. On the other hand, protein release of cytochrome $C(\mathrm{CytC})$, which is an inducer of apoptosis, from the intermembrane space was not observed. Celecoxib enhanced the membrane permeability of human erythrocytes and synthesized liposomes dose-dependently. It then induced the membrane-involving mitochondrial swelling and suppressed mitochondrial function.
\end{abstract}

Key words mitochondrial permeability transition; celecoxib; membrane

Mitochondria possess outer and inner membranes and produce energy in cells. The electron transfer system exists in the inner membrane and forms a proton gradient between the inside and outside of this membrane. ${ }^{1,2)}$ The proton gradient is important for the efficient production of ATP. In order to maintain this proton gradient, the permeability of the inner membrane is maintained at a low level, and, thus, most ions and molecules cannot pass through. Key protein (e.g. cytochrome $C$ ) that induces apoptosis and necrosis exists in the mitochondrial intermembrane space. ${ }^{3)}$ Mitochondria rapidly detect environmental signals, such as intercellular calcium concentration or oxidative stress, and release various proteins involved in the induction of cell death via a permeability change between the outer and inner membranes. The cell death-inducing mechanism involving a change in the permeability of mitochondrial membrane is called mitochondrial membrane permeability transition $(\mathrm{mPT}){ }^{4-6)} \mathrm{mPT}$ is induced by various exogenous factors $\left(e . g . \mathrm{Ca}^{2+}\right)$, and has been proposed as one of the target mechanisms of drug side effects.

Nonsteroidal anti-inflammatory drugs (NSAIDs) are widely used to treat a number of disorders associated with pain and inflammation. NSAIDs are known to cause adverse effects such as gastric ulceration and injury to the liver and kidneys. $^{7-11)}$ Although rare, NSAIDs have been reported to induce fulminant hepatitis. Certain NSAIDs impair cellular function by acting on mitochondria, and the relationship between hepatic damage and mitochondrial dysfunction (e.g. $\mathrm{mPT}$ ) has been examined. ${ }^{12-16)}$ In the present study, we investigated the effects of several NSAIDs on mitochondrial function, and observed celecoxib-induced mitochondrial swelling. Celecoxib is cyclooxygenase (COX)-2-selective NSAID that is widely used in the treatment of inflammation. We herein discussed cele- coxib-induced mitochondrial dysfunction (e.g. membrane permeability, energy synthesis, and membrane protein release).

\section{MATERIALS AND METHODS}

Reagents Celecoxib was purchased from LC Laboratories (Woburn, MA, U.S.A.). Mefenamic acid, fenbufen were purchased from Nacalai Tesque, Inc. (Kyoto, Japan). Naproxen, etodolac, salicylic acid, loxoprofen, acetylsalicylic acid, indomethacin, ibuprofen, piroxicam, and meloxicam were purchased from Wako Pure Chemical Industries, Ltd. (Osaka, Japan). Celecoxib, naproxen, etodolac, salicylic acid, loxoprofen, acetylsalicylic acid, indomethacin, and ibuprofen were dissolved in ethanol and stored at $-20^{\circ} \mathrm{C}$ until use. Mefenamic acid, fenbufen, piroxicam, and meloxicam were dissolved with dimethyl sulfoxide and stored at $-20^{\circ} \mathrm{C}$ until use. SF6847 (sc-200569) was purchased from Santa Cruz Biotechnology, Inc. (Santa Cruz, CA, U.S.A.). Cyclosporine A (CsA) was purchased from Nacalai Tesque, Inc. Anti-voltage dependent anion channel (VDAC) antibody were purchased from Cell Signaling Technology Japan (Tokyo, Japan). Anti-cytochrome $C$ (CytC) antibody and anti-adenylate kinase 2 (AK2) antibody were purchased from Signalway Antibody (Pearland, TX, U.S.A.) and Santa Cruz Biotechnology, Inc. (Santa Cruz, CA, U.S.A.), respectively. Anti-aldehyde dehydrogenase 2 (ALDH2) antibody was purchased from Abcam (Cambridge, U.K.). Anti-hydroxymethylglutaryl-CoA (HMG-CoA) synthase 2 (HMGCS2) antibody and anti-adenine nucleotide translocator 1 (ANT) antibody were kindly gifted from Professor Yasuo Shinohara (Tokushima University, Tokushima, Japan). Anti rabbit immunoglobulin G (IgG) of donkey origin (secondary antibody) was purchased from GE Healthcare Japan 
(Tokyo, Japan). The 1-palmitoyl-2-oleoylphosphatidylcholine (POPC) was purchased from Wako Pure Chemical Industries, Ltd. Calcein was purchased from Tokyo Chemical Industry Co., Ltd. (Tokyo, Japan).

Preparation of Rat Liver Mitochondria Preparation of liver mitochondria were performed as previously described from male Wistar rat. ${ }^{3,17)}$ Protein concentration of prepared mitochondrial suspension was examined by the Biuret method using bovine serum albumin as standard. All animal treatment was based on the animal care regulations of Suzuka University of Medical Science.

Swelling Analysis of Mitochondria Absorbance decreasing of mitochondrial suspension at $540 \mathrm{~nm}$ was examined at $25^{\circ} \mathrm{C}$ by spectrophotometric analysis using UV-1800 spectrophotometer (Shimadzu Co., Kyoto, Japan). ${ }^{18,19)}$ Mitochondria were incubated in inorganic phosphate (Pi)-containing medium (+Pi medium: sucrose (200 mM), K/Pi buffer (10 mm), $\mathrm{pH}$ $7.4)$ at $0.7 \mathrm{mg}$ protein $/ \mathrm{mL}$ of final concentration, and energized by succinate $(10 \mathrm{~mm})$ and rotenone $(1 \mu \mathrm{g} / \mathrm{mL})$. NSAIDs or $\mathrm{Ca}^{2+}$ $(100 \mu \mathrm{M})$ were added to the mitochondrial suspension, and the time-dependent absorbance change at $540 \mathrm{~nm}$ was examined.

Analysis of Mitochondrial Function Oxygen consumption rates (e.g. state4, state3, uncoupling) of mitochondria were examined using the $+\mathrm{Pi}$ medium as previously described. ${ }^{16)}$ Oxygen consumption rate was analyzed with a Clark type oxygen electrode (Yellow Spring 5331) as previously described method. ${ }^{18)}$

Analysis of Protein Release from Mitochondrial Compartments Mitochondria were incubated with $200 \mu \mathrm{M}$ celecoxib $\left(10 \mathrm{~min}, 25^{\circ} \mathrm{C}\right)$. After celecoxib treatment, the incubated solution were centrifuged $\left(13200 \mathrm{rpm}, 4^{\circ} \mathrm{C}, 5 \mathrm{~min}\right)$, and obtained the supernatant and precipitation. To evaluate the celecoxib-induced protein release from mitochondria, we subjected the mitochondrial lysates $(10.5 \mu \mathrm{g})$ to sodium dodecyl sulfate-polyacrylamide gel electrophoresis (SDS-PAGE) and subsequent Western analysis by using the first antibodies (antiVDAC, -CytC, -AK2, -ALDH2, -HMGCS2, and -ANT antibody). SDS-PAGE on 12.5 or $15.0 \%$ gel was performed as described by Laemmli. ${ }^{20)}$ Sample proteins that were obtained as described in the above were dissolved in $25 \mathrm{~mm}$ Tris- $\mathrm{HCl}(\mathrm{pH}$ 6.8) containing $1 \%$ SDS, $1 \%$ dithiothreitol and $10 \%$ glycerol and boiled for $5 \mathrm{~min}$ before electrophoresis. Western blotting was then performed as described by Towbin et al. ${ }^{21)}$ Horseradish peroxidase conjugated anti-rabbit IgG (donkey) was used as the second antibody. Detection was performed with ECL detection reagent (GE Healthcare).

Effect of Celecoxib on Lipid Membrane Human blood was obtained with informed consent (in accordance with Protocol No.15002, approved by the Institutional Ethical Review Board of the Institute of Technology and Science, Tokushima University Graduate School) from a healthy volunteer and stored in sterilized Alsever solution at $4^{\circ} \mathrm{C}$ until use. Stored blood for hemolysis assay was centrifuged $\left(3000 \mathrm{rpm}, 4^{\circ} \mathrm{C}\right.$, $3 \mathrm{~min}$ ) to remove the supernatant and buffy-coat. Erythrocytes were washed three times in phosphate buffered saline (PBS) (137 mm NaCl, $1.47 \mathrm{~mm} \mathrm{KH}_{2} \mathrm{PO}_{4}, 8.10 \mathrm{~mm} \mathrm{Na}_{2} \mathrm{HPO}_{4} 12 \mathrm{H}_{2} \mathrm{O}$, and $2.68 \mathrm{mM} \mathrm{KCl}$ ) with centrifugation. Washed erythrocytes were added at a final concentration of $0.5 \%(\mathrm{v} / \mathrm{v})$ to celecoxib, which was serially diluted with PBS. De-ionized water instead of celecoxib solution was used as a $100 \%$ hemolysis controls. After being incubated at $37^{\circ} \mathrm{C}$ for $90 \mathrm{~min}$, each mixture was centrifuged $\left(3000 \mathrm{rpm}, 4^{\circ} \mathrm{C}, 5 \mathrm{~min}\right)$ and the absorbance of the supernatant at $540 \mathrm{~nm}$ was assessed using the microplate reader Infinite M200 (TECAN, Mannedorf, Zurich, Switzerland). ${ }^{22)}$ Hemolytic activity was calculated as previously described. $^{23)}$

Calcein-loading Large Unilamellar Vesicles (Cal-LUVs) were prepared according to previously described methods. ${ }^{24)}$ A total of $7.6 \mathrm{mg}$ of POPC was dissolved in chloroform and dried by a nitrogen gas stream. Any remaining chloroform was completely removed under a vacuum for $2 \mathrm{~h}$. The lipid membrane that formed was hydrated with $1 \mathrm{~mL}$ calcein solution $(60 \mathrm{~mm}$ calcein, $200 \mathrm{~mm}$ sucrose, and $10 \mathrm{~mm} \mathrm{~K} / \mathrm{Pi}$ buffer, $\mathrm{pH}$ 7.4) for $30 \mathrm{~min}$. The hydrate became multilamellar vesicles (MLV) by vortexing. In order to prepare LUVs, MLV suspensions were extruded 11 times through a $100-\mathrm{nm}$ polycarbonate membrane filter using a mini-extruder (Avanti Polar Lipids, Alabaster, AL, U.S.A.) at $80^{\circ} \mathrm{C}$. Cal-LUV suspensions were passed through a Sephadex-G25 column twice to remove extravesicular calcein. The quantitative determination of prepared Cal-LUV was performed by the quantification of the phospholipid contents using LabAssay Phospholipid kit (Wako Pure Chemical Industries, Ltd.). Cal-LUVs were suspended in $+\mathrm{Pi}$ medium with the compound being tested (e.g. celecoxib) at $25^{\circ} \mathrm{C}$ for $60 \mathrm{~min}$, and $200 \mu \mathrm{L}$ of the suspension was added to a fluorescence assay black plate. Calcein release from CalLUVs was evaluated by fluorescence at $490 \mathrm{~nm}$ (Excitation) and $520 \mathrm{~nm}$ (Emission) using Gemini X-ray photoelectron spectroscopy (XPS) (Molecular Devices LLC., Sunnyvale, CA, U.S.A.).

\section{RESULTS}

NSAIDs Induced Mitochondrial Swelling The ability of various NSAIDs to induce $\mathrm{Ca}^{2+}$-like mitochondrial swelling was examined (Table 1). Celecoxib $(200 \mu \mathrm{M})$ induced similar mitochondrial swelling to $\mathrm{Ca}^{2+}(100 \mu \mathrm{M})$, and a decrease in absorbance $\left(\Delta \mathrm{Abs}_{540}\right)$ of $96.1 \%$ was observed. Mefenamic acid $(200 \mu \mathrm{M})$ also induced swelling and $\Delta \mathrm{Abs}_{540}$ was $72.7 \%$.

Table 1. NSAIDs Induced Mitochondrial Swelling

\begin{tabular}{lc}
\hline \hline & $\Delta \mathrm{Abs}_{540}(\%)$ \\
\hline $\mathrm{Ca}^{2+}$ & 100.0 \\
Celecoxib & $96.1 \pm 7.7$ \\
Mefenamic acid & $72.7 \pm 20.1$ \\
Fenbufen & $26.6 \pm 16.3$ \\
Diclofenac & $15.3 \pm 10.3$ \\
Etodolac & $9.1 \pm 9.4$ \\
Salicylic acid & $8.7 \pm 4.8$ \\
Loxoprofen & $5.1 \pm 5.7$ \\
Naproxen & $11.2 \pm 4.8$ \\
Acetylsalicylic acid & $4.3 \pm 0.3$ \\
Indomethacin & $6.7 \pm 1.3$ \\
Ibuprofen & $6.4 \pm 4.2$ \\
Piroxicam & $0.1 \pm 5.0$ \\
Meloxicam & $0.1 \pm 6.4$ \\
\hline
\end{tabular}

Mitochondrial swelling was examined as absorbance decrease at $540 \mathrm{~nm}\left(\Delta \mathrm{Abs}_{540}\right)$. After $\mathrm{Ca}^{2+}(100 \mu \mathrm{M})$ addition, the $\Delta \mathrm{Abs}_{540}$ during $10 \mathrm{~min}$ are shown as $100 \%$. Each drug $(200 \mu \mathrm{M})$ was added to mitochondria, and the $\Delta \mathrm{Abs}_{540}$ during $10 \mathrm{~min}$ was determined. The results are shown as the mean \pm standard deviation (S.D.) obtained from 3 independent experiments. 

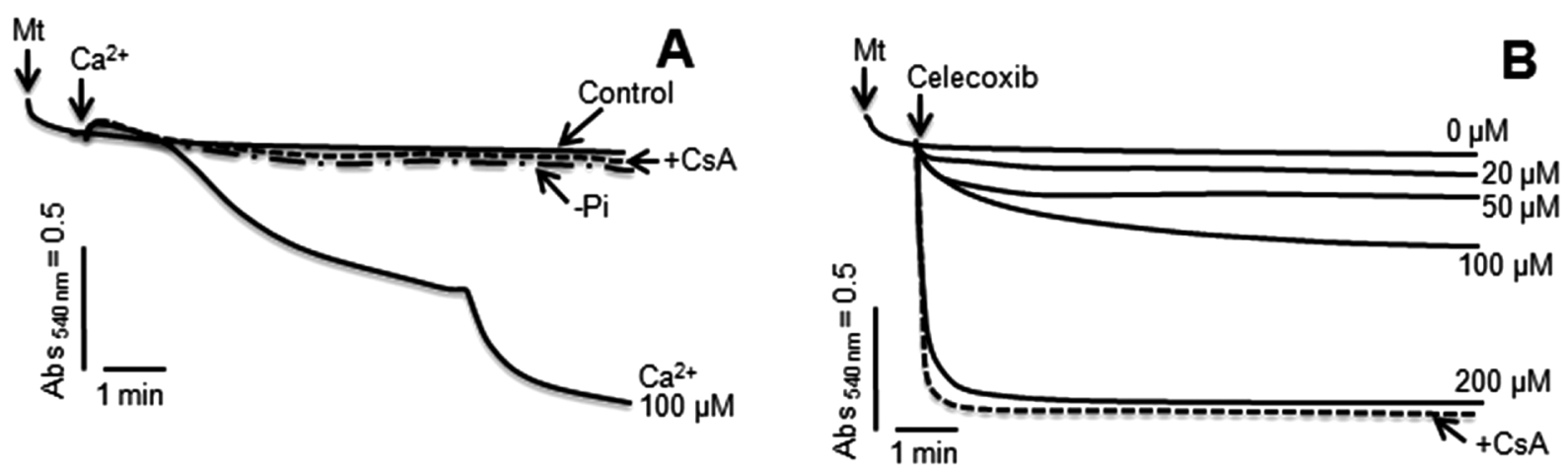

Fig. 1. Swelling Profiles of Mitochondria

Mitochondria (Mt) were suspended in $+\mathrm{Pi}$ medium $(0.7 \mathrm{mg} / \mathrm{mL}$ of final protein concentration) with succinate $(10 \mathrm{~mm})$ and rotenone $(1 \mu \mathrm{g} / \mathrm{mL})$ as respiratory substrates, and the absorbance change at $540 \mathrm{~nm}$ was monitored. After Mt addition, $100 \mu \mathrm{M} \mathrm{Ca}{ }^{2+}$ (A: solid line) and $20-200 \mu \mathrm{M}$ celecoxib (B: solid line) were supplied to reaction mixtures. Pi was omitted from medium, then evaluated the Pi effect (dashed line in Fig. 1A). CsA ( $1 \mu \mathrm{M})$ was added to medium, then assessed the CsA effect (dotted line in Figs. 1A, B).
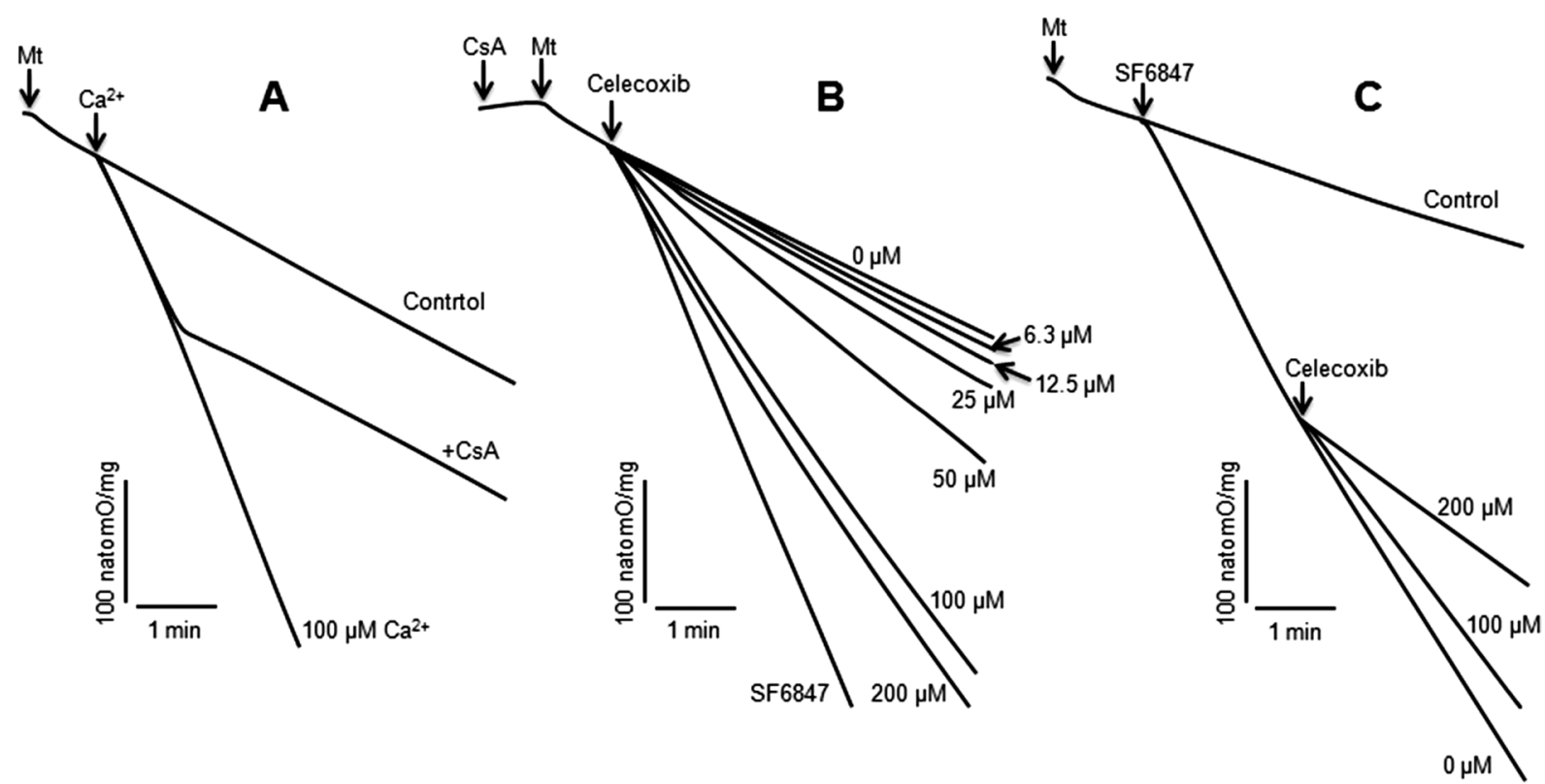

Fig. 2. Effect of Celecoxib on Mitochondrial Function

Mitochondrial oxygen consumption rates were examined, and evaluated the protonophoric effects of celecoxib. Panel A: effect of Ca ${ }^{2+}(100 \mu \mathrm{M})$ on oxygen consumption rate using the same condition as Fig. 1, and calcium-induced oxygen consumption was suppressed by CsA (1 $\mu \mathrm{M})$. Control line indicates the state 4 rate of oxygen consumption. Panel B: mitochondria were pretreated with CsA $(1 \mu \mathrm{M})$, then the facilitation of oxygen consumption by the induction of mPT was suppressed. Solid lines indicate the results, which were obtained using celecoxib $(6.3-200 \mu \mathrm{M})$ and SF6847 $(100 \mathrm{~nm})$ as a positive control of uncoupling. Panel C: effect of celecoxib on the $100 \mathrm{~nm}$ SF6847-induced uncoupling.

Other NSAIDs $(200 \mu \mathrm{m})$ tested in the present study exhibited a weak ability to induce mitochondrial swelling. Fenbufen $(26.6 \%)$, diclofenac $(15.3 \%)$, and naproxen (11.2\%) induced swelling. Etodolac, salicylic acid, loxoprofen, acetylsalicylic acid, indomethacin, and ibuprofen weakly induced swelling (5.1-9.1\%), while that induced by piroxicam and meloxicam was negligible.

The structure-activity relationship between mefenamic acid derivatives and mitochondrial function has already been reported. ${ }^{16)}$ In the present study, we examined the effects of celecoxib on mitochondrial function in more detail.

Effects of Celecoxib on Mitochondrial Swelling $\mathrm{Pi}$ is an essential element for calcium-induced $\mathrm{mPT}$, and $\mathrm{Ca}^{2+}$ $(100 \mu \mathrm{M})$ addition to mitochondrial suspensions induced the mitochondrial swelling, and decreased absorbance at $540 \mathrm{~nm}$ (solid line in Fig. 1A). In the absence of $\mathrm{Pi}, \mathrm{Ca}^{2+}$ addition did not markedly affect changes in absorbance (dashed line in Fig.
1A). CsA ( $1 \mu \mathrm{M})$ suppressed $\mathrm{Ca}^{2+}$-induced absorbance change completely (dotted line in Fig. 1A). Celecoxib $(20-200 \mu \mathrm{M})$ dose-dependently decreased absorbance at $540 \mathrm{~nm}$ (Fig. 1B). Celecoxib-induced decrease in absorbance were not suppressed by CsA (1 $\mu \mathrm{M})$ addition (dotted line in Fig. 1B). Thus, celecoxib exhibited discriminative feature for the modification of mitochondrial membranes.

Effects of Celecoxib on Mitochondrial Function Since the de-energization of mitochondria (e.g. uncoupling) had been shown to facilitate the mPT induction, ${ }^{14,25,26)}$ we examined the effects of $\mathrm{Ca}^{2+}$ and celecoxib on the oxygen consumption in mitochondria. The addition of $\mathrm{Ca}^{2+}(100 \mu \mathrm{M})$ induced uncoupling, and oxygen consumption increased markedly (Fig. 2A). This $\mathrm{Ca}^{2+}$-induced uncoupling was completely suppressed by the addition of CsA (1 $\mu \mathrm{M})$ (Fig. 2A).

In order to avoid the promotion of oxygen consumption by $\mathrm{mPT}$, mitochondria were pretreated with CsA $(1 \mu \mathrm{M})$, and un- 


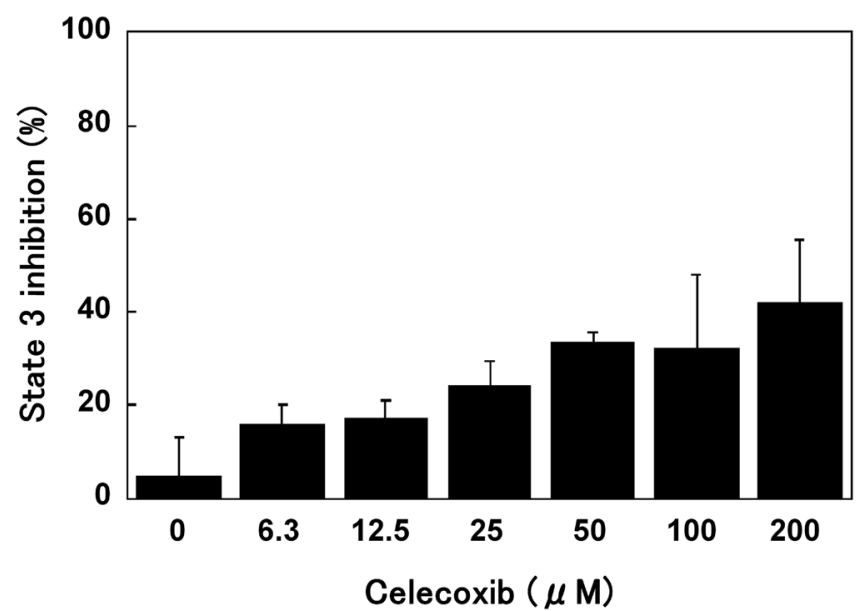

Fig. 3. Inhibition Effect of Celecoxib on the State 3 Oxygen Consumption

Mitochondria were suspended $(0.7 \mathrm{mg} / \mathrm{mL}$ of protein $)$ in $+\mathrm{Pi}$ medium with succinate $(10 \mathrm{~mm})$ and rotenone $(1 \mu \mathrm{g} / \mathrm{mL})$ as respiratory substrates, and state 3 oxygen consumption rate was examined with ADP $(1 \mathrm{~mm})$. Effect of celecoxib $(0-200 \mu \mathrm{M})$ on the oxygen consumption in ADP coexistent medium was monitored during $10 \mathrm{~min}$ as well as Fig. 2. The results of state 3 inhibition (\%) are shown as the mean \pm S.D. obtained from 3 independent experiments.

coupling states were examined. The rate of basal oxygen consumption (state 4$)$ was 32.3 natoms $/ \mathrm{mg} / \mathrm{min}(0 \mu \mathrm{M}$ celecoxib line in Fig. 2B), and increased to 151.6 natomsO/mg/min with SF6847 (100nM, positive control of uncoupling) (Fig. 2B). Celecoxib dose-dependently increased the oxygen consumption rate $(6.3-200 \mu \mathrm{M})$ (Fig. 2B). The oxygen consumption rate with $200 \mu \mathrm{M}$ celecoxib was 96.8 natomsO/mg/min, which was $54.0 \%$ of SF6847-induced uncoupling rate. Moreover, celecoxib inhibited SF6847-induced uncoupling, and the oxygen consumption rate decreased by 34.2 and $78.1 \%$ following the addition of 100 and $200 \mu \mathrm{M}$ celecoxib, respectively (Fig. 2C). These results indicate that celecoxib affects the mitochondrial electron transport system.

Energy synthesis is one of the key mitochondrial functions, and the state 3 rate is an index of ATP synthesis in mitochondria. Therefore, the inhibition ratio of celecoxib to the state 3 oxygen consumption rate was examined. As shown in Fig. 3 , celecoxib dose-dependently inhibited the state 3 rate. The state 3 rate was inhibited by $41.7 \%$ following the addition of $200 \mu \mathrm{M}$ celecoxib.

Effects of Celecoxib on Protein Release from Mitochondrial Compartments Celecoxib concentrations higher than $50 \mu \mathrm{M}$ increased protein release from mitochondria, and protein bands were detected using an SDS-PAGE analysis of supernatants (lanes 8-10 in Fig. 4B). Thus, mitochondrial component proteins were released by celecoxib-induced swelling.

VDAC (outer-membrane marker protein) was not released by celecoxib $(200 \mu \mathrm{M})$ and the VDAC band was only detected with precipitation (lane 3 in Fig. 4C versus 4D). CytC and $\mathrm{AK} 2$, intermembrane space marker proteins, were not detected in the supernatant following the addition of celecoxib (lane 3 in Fig. 4D), whereas ALDH2 and HMGCS2, matrix marker proteins, were present (lane 3 in Fig. 4D). HMGCS2 was fully released from the matrix compartment, and no bands were observed with precipitation (lane3 in Fig. 4C). The release of ANT (an inner-membrane marker protein) was not detected following the addition of celecoxib (lane 3 in Fig. 4D).
$\mathrm{Ca}^{2+}$, an inducer of mitochondrial swelling, released $\mathrm{CytC}$, $\mathrm{AK} 2, \mathrm{ALDH} 2$, and HMGCS2 at a concentration of $100 \mu \mathrm{M}$, and these bands were detected in the supernatant (lane 2 in Fig. 4D). $\mathrm{Ca}^{2+}$ released intermembrane space proteins (CytC and AK2) and the matrix proteins (ALDH2 and HMGCS2).

Effects of Celecoxib on Lipid Membranes The modifying effects of celecoxib on the permeability of lipid membranes were examined by a hemolytic analysis of human erythrocytes. Hemolysis was detected following the addition of celecoxib, with hemolysis ratios of $4.0 \%(50 \mu \mathrm{M}), 55.8 \%$ $(100 \mu \mathrm{M})$, and $93.6 \%(200 \mu \mathrm{M})$ being observed (Table 2).

In the permeability analysis of calcein including POPCliposomes, calcein release was detected following the addition of celecoxib. Calcein release ratios of 23.7, 79.3, and $90.1 \%$ were observed following the addition of 50,100 , and $200 \mu \mathrm{M}$ celecoxib, respectively (Table 2 )

\section{DISCUSSION}

NSAIDs are used as anti-inflammatory and anodyne medicine, but are associated with several adverse effects such as hepatopathy and gastrointestinal tract disturbances. ${ }^{7,9-11)} \mathrm{A}$ loss of liver mitochondrial function is considered as a factor of hepatopathy. ${ }^{27,28)} \mathrm{Ca}^{2+}$ induces mitochondrial swelling by formation of mitochondrial permeability transition (mPT) pore, and injures the mitochondrial function. ${ }^{4-6)}$ SF6847 disappears the $\mathrm{H}^{+}$gradient in mitochondrial inner membrane, and obstructs the mitochondrial function. We examined the effect of NSAIDs on mitochondria, and observed celecoxib-induced mitochondrial swelling (Table 1). The state of mitochondrial membrane proteins, which participate in the mitochondrial electron transport system, is altered by the mitochondrial swelling. Mitochondrial swelling leads to liver dysfunction.

A number of NSAIDs (e.g. meclofenamic acid, tolfenamic acid, flufenamic acid, diclofenac, and $N$-phenylanthranilic acid) have been shown to dose-dependently induce mitochondrial swelling. ${ }^{12,16)}$ Their induction of mitochondrial swelling was suppressed by CsA $(1 \mu \mathrm{M})$. Various NSAIDs (e.g. $200 \mu \mathrm{M}$ piroxicam, meloxicam in Table 1) exhibited no swelling effect on mitochondria, and it is difficult to think that mitochondrial swelling depends only on the osmotic pressure of NSAID. In the present study, celecoxib-induced mitochondrial swelling occurred in a dose-dependent manner up to $200 \mu \mathrm{M}$ and was not suppressed by CsA (Fig. 1). These results indicate that celecoxib-induced swelling involves a different mechanism to that of calcium-induced swelling. Induction effect of mitochondrial swelling by mefenamic acid has been reported, and the structure of pharmacophore, which participates in mitochondrial function, was previously reported. ${ }^{16)}$

A relation between the activity balance of COX-1 and COX-2 and the side effect of NSAIDs (e.g. hepatopathy and gastroenteropathy) have been examined..$^{9,10,29,30)}$ Since gastroenteropathy occur when COX-1 is strongly obstructed, medication that selectively obstructs COX-2 has been developed. COX-2-selective celecoxib dose-dependently exerts uncoupling effects on mitochondria, similar to SF6847 (Figs. 2A, B). However, celecoxib-induced uncoupling is not suppressed by $\mathrm{CsA}$, and, thus, celecoxib modifies mitochondrial function via a different mechanism to $\mathrm{Ca}^{2+}$. Moreover, celecoxib dose-dependently restrained the uncoupling effects of SF6847, which suggests that it also obstructs the electron transfer 

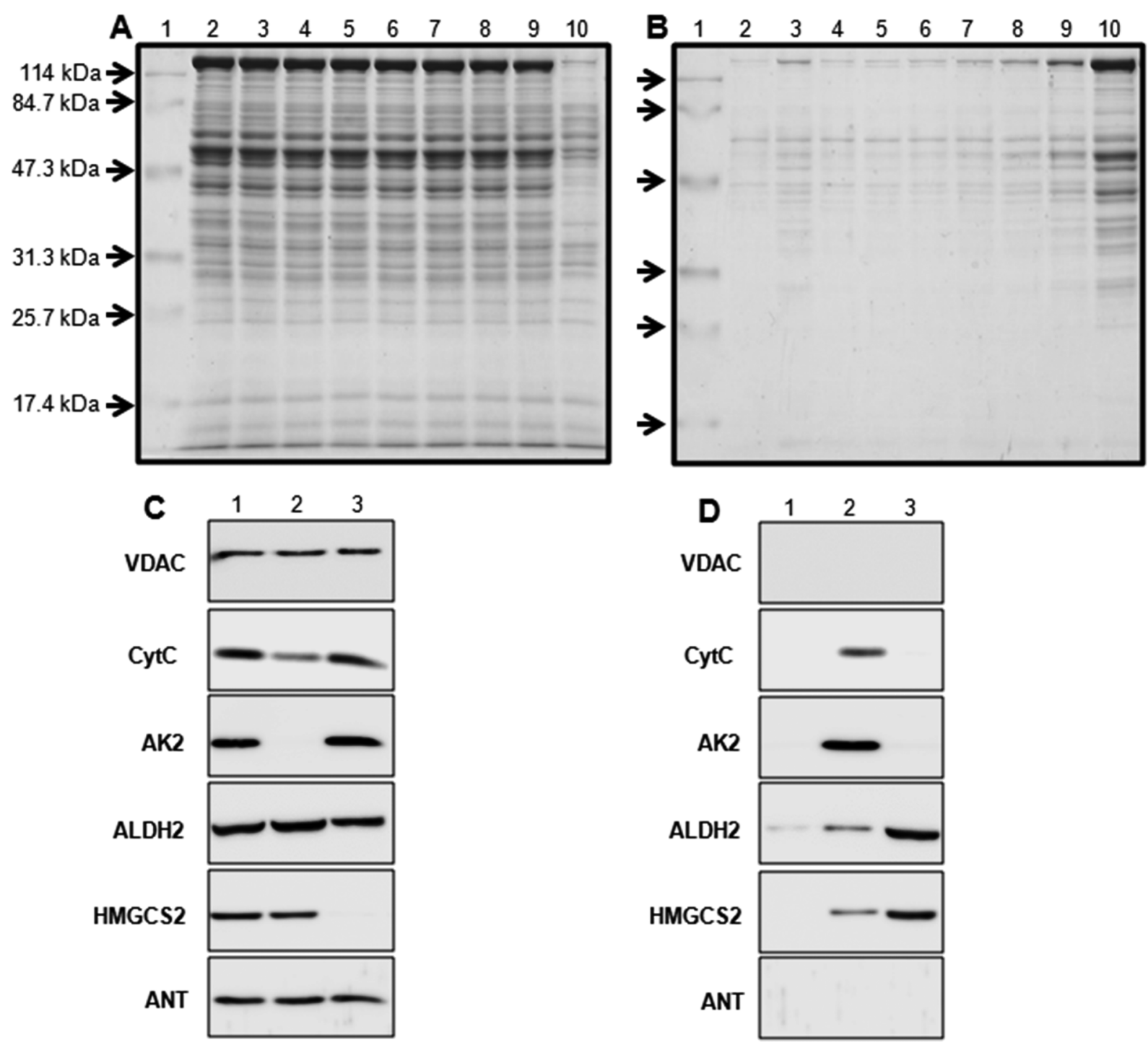

Fig. 4. Protein Release Profile from Mitochondrial Compartment

SDS-PAGE and Western blotting analysis was performed with celecoxib treated mitochondria (Figs. 4A, C: precipitation, 4B, 4D: supernatant). Samples were prepared as the same experimental condition as Fig. 1. Lanes 1-10 in Figs. 4A and B indicated as follows: molecular maker proteins (lane 1), intact mitochondrial extract (lane 2), $100 \mu \mathrm{M}$ calcium treated (lane 3) and celecoxib treated samples ((3.1 $\mu \mathrm{M}$ (lane 4), 6.3 $\mu \mathrm{m}$ (lane 5), 12.5 $\mu \mathrm{M}$ (lane 6), 25 $\mu \mathrm{M}$ (lane 7), 50 $\mu \mathrm{M}$ (lane 8), 100 $\mu \mathrm{M}$ (lane 9), and 200 $\mu \mathrm{M}$ (lane 10)). Released proteins were detected by anti-VDAC, anti-CytC, anti-AK2, anti-ALDH2, anti-HMGCS2, and anti-ANT antibody (Figs. 4C, D). Lanes 1-3 in Figs. 4C and D indicated as follows: intact mitochondrial extract (lane 1), $100 \mu \mathrm{M}$ calcium treated (lane 2) and 200 $\mu \mathrm{M}$ celecoxib treated sample (lane 3).

Table 2. Effect of Celecoxib on the Permeability of Lipid Membrane

\begin{tabular}{ccc}
\hline \hline Celecoxib $(\mu \mathrm{M})$ & Erythrocyte $(\%)$ & POPC-liposome $(\%)$ \\
\hline 0 & $0.0 \pm 0.0$ & $0.0 \pm 0.0$ \\
50 & $4.0 \pm 0.7$ & $23.7 \pm 1.5$ \\
100 & $55.8 \pm 3.4$ & $79.3 \pm 4.6$ \\
200 & $93.6 \pm 1.9$ & $90.1 \pm 4.3$
\end{tabular}

The $100 \%$ lysis (control) of tested membrane was obtained by using de-ionized water (erythrocyte) and $1 \%$ triton X-100 (POPC-liposome). The membrane lysis ratio (\%) to control was determined. Results are shown as the mean \pm S.D. obtained from 3 independent experiments.

system of mitochondria (Fig. 2C). SF6847 disappears the $\mathrm{H}^{+}$ gradient of mitochondrial inner membrane and indicates the biggest oxygen consumption rate. This oxygen consumption is including the oxygen consumption by an electron transfer system. An electron transfer system was obstructed by celecoxib, and SF6847 induced oxygen consumption rate decreased by celecoxib addition. Celecoxib inhibited state 3, which involves ATP synthesis in the mitochondrial inner membrane (Fig. 3), and, thus, may suppress mitochondrial function. Celecoxib has been suggested to modify membrane conditions (e.g. fluidity), and exert at least 2 of the following effects: 1 ; the induction of uncoupling actions through a change in the membrane permeability of protons, and 2; the induction of irregularities in the electron transfer system through functional modifications to inner membrane-localized proteins involved in electron transfer. Since a decrease in absorbance (mitochondrial swelling) was not observed with SF6847 (data not shown), celecoxib appears to use a different mechanism of action to SF6847.

Celecoxib-induced protein release from mitochondrial compartment (the outer membrane, inner membrane, intermembrane space, and matrix) was also investigated in the present study, and the isolation of matrix proteins (e.g. ALDH2 and HMGCS2) was confirmed (Fig. 4). The degree of release of these celecoxib-induced matrix proteins was greater than 
calcium-induced protein release, indicating that mitochondrial morphology is strongly modified (e.g. aggregation) by the addition of celecoxib. The release of marker proteins from the outer membrane, inner membrane, and intermembrane space was not observed following the addition of celecoxib. Celecoxib produced a unique permeability system that differed from calcium-induced mPT. The release of CytC was not observed following the addition of celecoxib, suggesting that celecoxib did not induce $\mathrm{Ca}^{2+}$-like apoptosis.

Celecoxib caused hemolysis in a dose-dependent manner, with $93.6 \%$ of erythrocytes being lysed by the addition of $200 \mu \mathrm{M}$ celecoxib (Table 2). Celecoxib also induced the lysis of POPC-liposomes, with $90 \%$ of liposomes being lysed by $200 \mu \mathrm{M}$ celecoxib. Celecoxib appears to recognize the general constituents of membranes (e.g. phospholipid) and modifies membrane features (e.g. fluidity). Mitochondrial membranes (particularly the inner membrane) contain many types of proteins (e.g. electron transporters and substrate transporters), and differ physically from general phospholipid cell membranes. After the binding of celecoxib to the mitochondrial membrane, the mitochondrial structure seemed to be maintained temporarily. However, the matrix proteins may have been released through the opening of the mPT pore-like system. The effects of pharmaceuticals, which cause hepatopathy as a side effect, on mitochondrial function are being investigated. The relation between COX-1, COX-2 selectivity and mitochondrial dysfunction (e.g. swelling) is the subject of future investigation. The analysis of mitochondrial function may not be related to a clinical event directly, but the mitochondria associated examination (e.g. NSAID induced swelling) should be useful for understanding the feature of drugs.

Acknowledgment This work was supported, in part, by a Grant-in-Aid for Scientific Research (15K08113) from the Ministry of Education, Culture, Sports, Science and Technology of the Japanese Government.

Conflict of Interest The authors declare no conflict of interest.

\section{REFERENCES}

1) Mitchell P. Coupling of phosphorylation to electron and hydrogen transfer by a chemi-osmotic type of mechanism. Nature, 191, 144-148 (1961).

2) Kadenbach B, Ramzan R, Wen L, Vogt S. New extension of the Mitchell Theory for oxidative phosphorylation in mitochondria of living organisms. Biochim. Biophys. Acta, 1800, 205-212 (2010).

3) Yamada A, Yamamoto T, Yamazaki N, Yamashita K, Kataoka M, Nagata $\mathrm{T}$, Terada $\mathrm{H}$, Shinohara Y. Differential permeabilization effects of $\mathrm{Ca}^{2+}$ and valinomycin on the inner and outer mitochondrial membranes as revealed by proteomics analysis of proteins released from mitochondria. Mol. Cell. Proteomics, 8, 1265-1277 (2009).

4) Nakagawa T, Shimizu S, Watanabe T, Yamaguchi O, Otsu K, Yamagata $\mathrm{H}$, Inohara $\mathrm{H}$, Kubo T, Tsujimoto Y. Cyclophilin D-dependent mitochondrial permeability transition regulates some necrotic but not apoptotic cell death. Nature, 434, 652-658 (2005).

5) Tsujimoto Y, Shimizu S. Role of the mitochondrial membrane permeability transition in cell death. Apoptosis, 12, 835-840 (2007).

6) Biasutto L, Azzolini M, Szabò I, Zoratti M. The mitochondrial permeability transition pore in AD 2016: An update. Biochim. Biophys.
Acta, 1863, 2515-2530 (2016).

7) Graham DY, Opekun AR, Willingham FF, Qureshi WA. Visible small-intestinal mucosal injury in chronic NSAID users. Clin. Gastroenterol. Hepatol., 3, 55-59 (2005).

8) Musu M, Finco G, Antonucci R, Polati E, Sanna D, Evangelista M, Ribuffo D, Schweiger V, Fanos V. Acute nephrotoxicity of NSAID from the foetus to the adult. Eur. Rev. Med. Pharmacol. Sci., 15, 1461-1472 (2011).

9) Harirforoosh S, Asghar W, Jamali F. Adverse effects of nonsteroidal antiinflammatory drugs: an update of gastrointestinal, cardiovascular and renal complications. J. Pharm. Pharm. Sci., 16, 821-847 (2013).

10) Bessone F. Non-steroidal anti-inflammatory drugs: What is the actual risk of liver damage? World J. Gastroenterol., 16, 5651-5661 (2010).

11) Unzueta A, Vargas HE. Nonsteroidal anti-inflammatory druginduced hepatoxicity. Clin. Liver Dis., 17, 643-656, ix (2013).

12) Masubuchi Y, Yamada S, Horie T. Possible mechanism of hepatocyte injury induced by diphenylamine and its structurally related nonsteroidal anti-inflammatory drugs. J. Pharmacol. Exp. Ther., 292, 982-987 (2000).

13) Berson A, Cazanave S, Descatoire V, Tinel M, Grodet A, Wolf C, Feldmann G, Pessayre D. The anti-inflammatory drug, nimesulide (4-nitro-2-phenoxymethane-sulfoanilide), uncouples mitochondria and induces mitochondrial permeability transition in human hepatoma cells: protection by albumin. J. Pharmacol. Exp. Ther., 318, 444-454 (2006).

14) Lal N, Kumar J, Erdahl WE, Pfeiffer DR, Gadd ME, Graff G, Yanni JM. Differential effects of non-steroidal anti-inflammatory drugs on mitochondrial dysfunction during oxidative stress. Arch. Biochem. Biophys., 490, 1-8 (2009).

15) Syed M, Skonberg C, Hansen SH. Mitochondrial toxicity of selective COX-2 inhibitors via inhibition of oxidative phosphorylation (ATP synthesis) in rat liver mitochondria. Toxicol. In Vitro, 32, 26-40 (2016).

16) Tatematsu Y, Hayashi H, Taguchi R, Fujita H, Yamamoto A, Ohkura K. Effect of $N$-phenylanthranilic acid scaffold nonsteroidal anti-inflammatory drugs on the mitochondrial permeability transition. Biol. Pharm. Bull., 39, 278-284 (2016).

17) Kawashima S, Yamamoto $T$, Horiuchi $Y$, Fujiwara $K$, Gouda S, Yoshimura Y, Yamamoto A, Inotani Y, Yamashita K, Kitamura S, Terada H, Kanematsu M, Shishido K, Shinohara Y. S-15176 and its methylated derivative suppress the CsA-insensitive mitochondrial permeability transition and subsequent cytochrome $c$ release induced by silver ion, and show weak protonophoric activity. Mol. Cell. Biochem., 358, 45-51 (2011).

18) Shinohara Y, Almofti MR, Yamamoto $T$, Ishida T, Kita F, Kanzaki H, Ohnishi M, Yamashita K, Shimizu S, Terada H. Permeability transition-independent release of mitochondrial cytochrome $c$ induced by valinomycin. Eur. J. Biochem., 269, 5224-5230 (2002).

19) Yamamoto T, Yoshimura Y, Yamada A, Gouda S, Yamashita K, Yamazaki N, Kataoka M, Nagata T, Terada H, Shinohara Y. Distinct behaviors of adenylate kinase and cytochrome $c$ observed following induction of mitochondrial permeability transition by $\mathrm{Ca}^{2+}$ in the absence of respiratory substrate. J. Bioenerg. Biomembr., 40, 619-623 (2008).

20) Laemmli UK. Cleavage of structural proteins during the assembly of the head of bacteriophage T4. Nature, 227, 680-685 (1970).

21) Towbin H, Staehelin T, Gordon J. Electrophoretic transfer of proteins from polyacrylamide gels to nitrocellulose sheets: procedure and some applications. Proc. Natl. Acad. Sci. U.S.A., 76, 4350-4354 (1979)

22) Tabata A, Ohkura K, Ohkubo Y, Tomoyasu T, Ohkuni H, Whiley RA, Nagamune H. The diversity of receptor recognition in cholesterol-dependent cytolysins. Microbiol. Immunol., 58, 155-171 (2014). 
23) Nagamune H, Ohnishi C, Katsuura A, Fushitani K, Whiley RA, Tsuji A, Matsuda Y. Intermedilysin, a novel cytotoxin specific for human cells secreted by Streptococcus intermedius UNS46 isolated from a human liver abscess. Infect. Immun., 64, 3093-3100 (1996).

24) Yamamoto $T$, Ito $M$, Kageyama $K$, Kuwahara $K$, Yamashita $K$, Takiguchi Y, Kitamura S, Terada H, Shinohara Y. Mastoparan peptide causes mitochondrial permeability transition not by interacting with specific membrane proteins but by interacting with the phospholipid phase. FEBS J., 281, 3933-3944 (2014).

25) Petronilli V, Cola C, Bernardi P. Modulation of the mitochondrial cyclosporin A-sensitive permeability transition pore. II. The minimal requirements for pore induction underscore a key role for transmembrane electrical potential, matrix $\mathrm{pH}$, and matrix $\mathrm{Ca}^{2+}$. J. Biol. Chem., 268, 1011-1016 (1993).

26) Beatrice MC, Palmer JW, Pfeiffer DR. The relationship between mitochondrial membrane permeability, membrane potential, and the retention of $\mathrm{Ca}^{2+}$ by mitochondria. J. Biol. Chem., 255, 8663-8671 (1980).

27) Pessayre D, Fromenty B, Berson A, Robin MA, Lettéron P, Moreau $\mathrm{R}$, Mansouri A. Central role of mitochondria in drug-induced liver injury. Drug Metab. Rev., 44, 34-87 (2012).

28) Vuda M, Kamath A. Drug induced mitochondrial dysfunction: Mechanisms and adverse clinical consequences. Mitochondrion, 31, 63-74 (2016).

29) Jones P, Lamdin R. Oral cyclo-oxygenase 2 inhibitors versus other oral analgesics for acute soft tissue injury: systematic review and meta-analysis. Clin. Drug Investig., 30, 419-437 (2010).

30) Souto EO, Miyoshi H, Dubois RN, Gores GJ. Kupffer cell-derived cyclooxygenase-2 regulates hepatocyte $\mathrm{Bcl}-2$ expression in choledocho-venous fistula rats. Am. J. Physiol. Gastrointest. Liver Physiol., 280, G805-G811 (2001). 